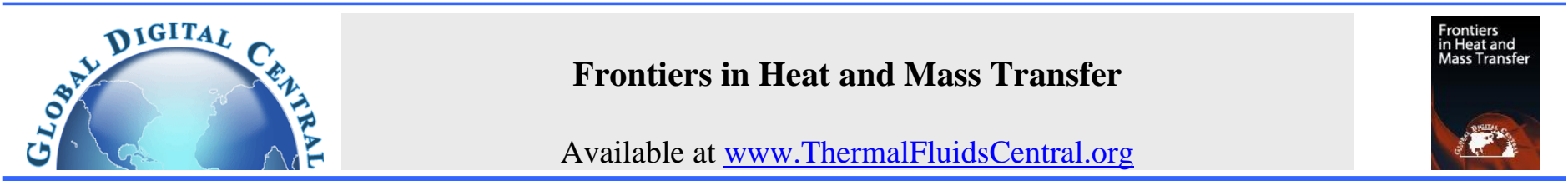

\title{
INFLUENCE OF CONVECTIVE BOUNDARY CONDITION ON NONLINEAR THERMAL CONVECTION FLOW OF A MICROPOLAR FLUID SATURATED POROUS MEDIUM WITH HOMOGENEOUS-HETEROGENEOUS REACTIONS
}

\author{
Chetteti RamReddy ${ }^{\mathrm{a} \dagger}$ and Teegala Pradeepa ${ }^{\mathrm{a}}$

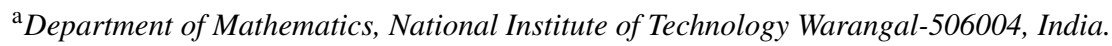

\begin{abstract}
A numerical approach has been used to analyze the effects of homogeneous-heterogeneous reaction and nonlinear density temperature variation over a vertical plate in an incompressible micropolar fluid flow saturated Darcy porous medium. In addition, convective boundary condition is incorporated in a micropolar fluid model. The similarity representation for the set of partial differential equations is attained by applying Lie group transformations. The resulting non-dimensional equations are worked out numerically by spectral quasi-linearization method. Less temperature and wall couple stress coefficient, but more velocity, skin friction, species concentration, and heat transfer rate are noticed by enhancing the nonlinear convection parameter. It is also observed that as the strength of homogeneous and heterogeneous reaction parameters increases, the species concentration and mass transfer rate reduces.

Keywords: Non-linear convection, Convective boundary condition, Micropolar fluid, Homogeneous-heterogeneous reactions, Porous medium, Spectral quasi-linearization method.
\end{abstract}

\section{INTRODUCTION}

The analysis of convective transport in porous media has been a topic of continuing interest in the past decades due to its significance in various engineering, scientific and industrial applications like air conditioning of a room, moisture transport in thermal insulation, solar energy collecting devices, petroleum industries, material processing, cooling of molten metals, etc. Darcy's law is a linear empirical relationship between the Darcian velocity and the pressure drop across the porous medium and it is valid for slow flows with low permeability in porous media. The literary work relevant to the convective heat and mass transfer phenomenon in porous medium can be found in the books by Vafai (2000); Ingham and Pop (2005); Nield and Bejan (2013). Many researchers combined these analysis with non-Newtonian fluids, but most of them cannot explain the rotation of fluid particles and its rheological behaviour. Thus, Eringen (1966) introduced the micropolar fluids subset of micromorphic fluid theory, and they exhibit the microrotation and micro-inertia effects. Further, they simulate the flow attributes of animal blood, haematological suspensions, geomorphological sediments, liquid crystal, colloidal suspensions etc. A comprehensive review of theory and applications of micropolar fluids can be presented in the books by (Lukaszewicz, 1999; Eremeyev et al., 2013). The analysis of free convection flow of micropolar fluids in porous medium has been investigated by many researchers with different analysis, (Singh, 2013; El-Kabeir and Gorla, 2007; Beg et al., 2007;
Srinivasacharya and RamReddy, 2013; Pal and Chatterjee, 2015), etc.

Several investigators extended their work with chemical reactions due to its immense practical applications in engineering and science. Chemical reactions can be divided as homogeneous and heterogeneous reactions. Homogeneous reaction occurs uniformly throughout a given phase while heterogeneous reactions arises in a particular area or at phase boundaries. So, these reactions depend on phase interface. The influence of homogeneous and heterogeneous reactions in chemically reacting system arises in catalysis, combustion and bio-chemical system. Merkin (1996) discussed homogeneous-heterogeneous reaction model in boundary layer flow in which cubic autocatalysis represents the homogeneous reaction and the heterogeneous reaction by a first-order process. The effect of homogeneous-heterogeneous reaction in a nanofluid flow towards a porous shrinking/stretching sheet has been investigated by (Kameswaran et al., 2013). (Shaw et al., 2013) analyzed the incompressible micropolar fluid flow over a stretching or shrinking sheet with the influence of permeability and homogeneous-heterogeneous reactions. (Nandkeolyar et al., 2014) studied the effects of external magnetic field, internal heat generation and homogeneous-heterogenous reaction on water based nanofluid flow over a stretching surface. (Hayat et al., 2015a) analyzed stagnation point flow of carbon nanotubes induced by an impermeable stretching cylinder with homogeneous-heterogeneous reactions and Newtonian

${ }^{\dagger}$ Corresponding author: chramreddy@nitw.ac.in; chittetiram@gmail.com 
heating effects. The effect of Cattaneo-Christov heat flux in the stagnation point flow of Maxwell fluid towards a nonlinear stretching surface with homogeneous-heterogeneous reactions has been examined by (Hayat et al., 2016a).

The influence of nonlinear density temperature in the buoyancy force term may exert a variation in flow and heat transfer characteristics owing to the temperature difference between the ambient fluid and surface of the plate becomes consequently large. The physical significance of nonlinear thermal convection is of great interest owing to a wide range of applications in astrophysics, engineering, geophysics and industrial manufacturing process such as doping processes, cooling of electronic components, pore water convection near salt domes etc. Barrow and Rao (1971) discussed the variable thermal expansion coefficient effect on free convection flow. The study of natural convection flow embedded in a non-Darcy porous medium with a temperature-concentration-dependent density relation has been studied by Partha (2010). (Kameswaran et al., 2014) presented the effect of thermophoresis on nonlinear convection flow over an impermeable vertical wall in a Darcy porous medium. (Motsa et al., 2014a) discussed the unsteady nonlinear convective flow caused by an impulsive stretching sheet, and generated the numerical results with spectral homotopy analysis method. (Shaw et al., 2016) studied the nonlinear convection flow of nanofluid over a stretched surface with the effect of momentum, thermal, and solute slip.

Most of the researchers showed attention to a great extent on heat transfer problems related to the convective surface boundary condition. It is more general and realistic, especially in various technologies and industrial operations such as material drying, transpiration, laser pulse heating, current carrying conductor cooled by ambient air, nuclear fuel rod cooled by a liquid metal coolant etc[see (Hayat et al., 2015b, 2016c,d)]. The hydro magnetic boundary layer flow with heat and mass transfer over a vertical plate in the presence of magnetic field and chemical reaction with the convective boundary condition is scrutinized by (Gangadhar et al., 2012). Yacob and Ishak (2012) investigated stagnation point flow towards stretching or shrinking sheet in an incompressible micropolar fluid using convective boundary condition and presented dual solutions for shrinking case but, for stretching case the solution is unique. (Hayat et al., 2013) discussed the influence of convective boundary condition on mixed convection stagnation flow of Maxwell fluid with radiation. (Hayat et al., 2014) analyzed the effects of Joule heating and thermophoresis in stagnation point flow of Maxwell fluid with convective condition. (Chamka et al., 2014) studied the effects of viscous dissipation and magnetic field on free convection flow in a non Darcy porous medium saturated with a nanofluid under convective boundary condition. (RamReddy et al., 2015) analyzed the similarity solution of free convection flow of an incompressible micropolar fluid with convective boundary condition using lie group transformations. (Waqas et al., 2016) investigated the influence of convective boundary condition on MHD mixed convection flow of micropolar fluid towards nonlinear stretched sheet. Micropolar fluid flow and heat transfer over an unsteady stretching sheet subject to convective boundary condition has been discussed by (Shehzad1 et al., 2016). (Hayat et al., 2016b) studied the MHD flow of third grade fluid due to exponentially stretching sheet with convective boundary condition.

From these works and literature survey, it conclude that the nonlinear convection flow of micropolar fluid saturated Darcy porous medium in the presence of homogeneous-heterogeneous reactions using convective boundary condition have not been investigated so far. This article attempts to present the new similarity transformations and corresponding similarity solution to the homogeneous-heterogeneous effect on natural convection flow of a micropolar fluid saturated Darcy porous medium under convective boundary condition in the presence of nonlinear dependent temperature relation. The involvement of convective boundary conditions for mathematical model becomes a slightly more complicated leading to the complex interactions of the flow and heat transfer mechanism. Fur- ther, numerical solution is obtained since the analytical solution is out of scope for the current problem. Also, the influence important parameters, namely, convective heat transfer, nonlinear convection, strength of homogeneous and heterogeneous reaction parameters on the physical quantities of the flow, heat transfer rates are analyzed in different flow situations.

\section{MATHEMATICAL FORMULATION}

Consider the steady, two dimensional, laminar and incompressible micropolar fluid flow along a vertical plate embedded in Darcy porous medium. The fluid flow is influenced by nonlinear convection due to nonlinear temperature and density relation. The $\bar{x}$-axis is chosen along the vertical plate and $\bar{y}$-axis is normal to the plate, as shown in Fig. (1). The free stream temperature is $T_{\infty}$. Due to the convection, the plate is either cooled/heated from a fluid of temperature $T_{f}$ with $T_{f}<T_{\infty}$ related to a cooled surface (opposing flow) and $T_{f}>T_{\infty}$ corresponding to a heated surface (assisting flow) and respectively.

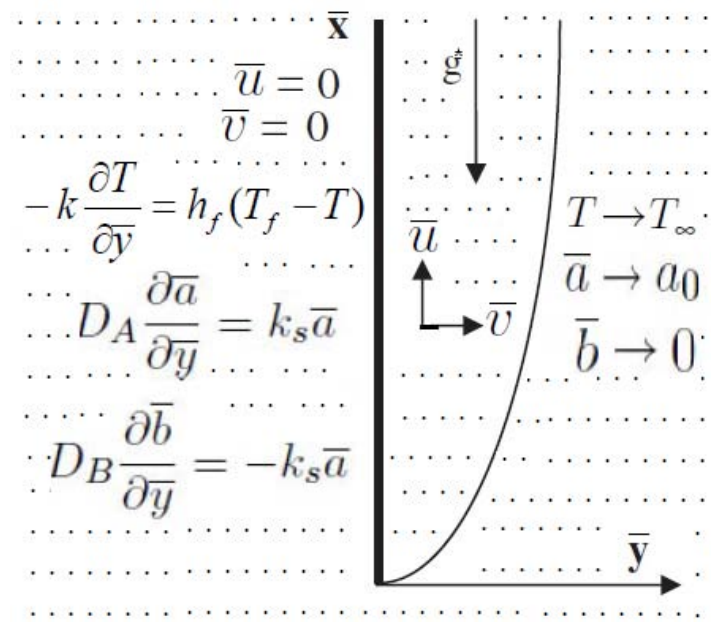

Fig. 1 Geometry of the Problem

Further, the following assumptions are considered in the present analysis: (i) The porous medium is homogeneous and isotropic,(i.e., uniform with a constant porosity and permeability).

(ii) Except for the density variation prescribe by the Boussinesq approximation, the porous medium and fluid properties are constant.

(iii) The porous medium and fluid are in local thermodynamic equilibrium.

(iv) A simple homogeneous-heterogeneous reaction model exists as considered by Merkin (1996) in the following form :

$$
A+2 B \rightarrow 3 B, \quad \text { rate }=k_{c} \bar{a} \bar{b}^{2}
$$

The single isothermal first order reaction on the catalyst surface

$$
A \rightarrow B, \quad \text { rate }=k_{s} \bar{a}
$$

By employing these conditions and making use of the standard boundary layer approximations, the governing equations for the micropolar fluid Ahmadi (1976) are given by

$$
\begin{gathered}
\frac{\partial \bar{u}}{\partial \bar{x}}+\frac{\partial \bar{v}}{\partial \bar{y}}=0 \\
\frac{\rho}{\epsilon^{2}}\left(\bar{u} \frac{\partial \bar{u}}{\partial \bar{x}}+\bar{v} \frac{\partial \bar{u}}{\partial \bar{y}}\right)=\frac{1}{\epsilon}(\mu+\kappa) \frac{\partial^{2} \bar{u}}{\partial \bar{y}^{2}}+\kappa \frac{\partial \bar{\omega}}{\partial \bar{y}}-\frac{\mu}{K_{p}} \bar{u} \\
+\rho g^{*}\left[\beta_{1}\left(T-T_{\infty}\right)+\beta_{2}\left(T-T_{\infty}\right)^{2}\right] \\
\frac{\rho j}{\epsilon}\left(\bar{u} \frac{\partial \bar{\omega}}{\partial \bar{x}}+\bar{v} \frac{\partial \bar{\omega}}{\partial \bar{y}}\right)=\gamma \frac{\partial^{2} \bar{\omega}}{\partial \bar{y}^{2}}-\kappa\left(2 \bar{\omega}+\frac{1}{\epsilon} \frac{\partial \bar{u}}{\partial \bar{y}}\right)
\end{gathered}
$$




$$
\begin{gathered}
\bar{u} \frac{\partial T}{\partial \bar{x}}+\bar{v} \frac{\partial T}{\partial \bar{y}}=\alpha \frac{\partial^{2} T}{\partial \bar{y}^{2}} \\
\bar{u} \frac{\partial \bar{a}}{\partial \bar{x}}+\bar{v} \frac{\partial \bar{a}}{\partial \bar{y}}=D_{A} \frac{\partial^{2} \bar{a}}{\partial \bar{y}^{2}}-k_{c} \bar{a}^{2} \\
\bar{u} \frac{\partial \bar{b}}{\partial \bar{x}}+\bar{v} \frac{\partial \bar{b}}{\partial \bar{y}}=D_{B} \frac{\partial^{2} \bar{b}}{\partial \bar{y}^{2}}+k_{c} \bar{a} \bar{b}^{2}
\end{gathered}
$$

where $\bar{\omega}$ represents the microrotation component, $\bar{u}$ and $\bar{v}$ are components of Darcy velocity in $\bar{x}$ and $\bar{y}$ directions respectively, $\kappa$ is the vortex viscosity, $\bar{a}, \bar{b}$ are concentrations of the chemical species A and B, $\rho$ represents the fluid density, $T$ indicates the temperature, $D_{A}$ and $D_{B}$ are the respective diffusion coefficients of species $\mathrm{A}$ and $\mathrm{B}, g^{*}$ represents the acceleration due to gravity, $K_{p}$, is the permeability, $\mu$ indicates the dynamic coefficient of viscosity, $\beta_{1}, \beta_{2}$ are the coefficient of thermal expansion, $j$ is the micro-inertia density, $\alpha$ is the thermal diffusivity and $\gamma$ is the spingradient viscosity.

The boundary conditions are

$$
\begin{gathered}
\bar{u}=0, \quad \bar{v}=0, \quad \bar{\omega}=-n \frac{\partial \bar{u}}{\partial \bar{y}}, \quad-k \frac{\partial T}{\partial \bar{y}}=h_{f}\left(T_{f}-T\right), \\
D_{A} \frac{\partial \bar{a}}{\partial \bar{y}}=k_{s} \bar{a}, \quad D_{B} \frac{\partial \bar{b}}{\partial \bar{y}}=-k_{s} \bar{a} \quad \text { at } \quad \bar{y}=0 \\
\bar{u}=0, \quad \bar{\omega}=0, \quad T=T_{\infty}, \quad \bar{a}=a_{0}, \quad \bar{b}=0 \quad \text { as } \quad \bar{y} \rightarrow \infty
\end{gathered}
$$

where, the subscripts $w$ and $\infty$ indicate the conditions at the wall and at the outer edge of the boundary layer respectively, $h_{f}$ is the convective heat transfer coefficient, $k$ is the thermal conductivity of the fluid, $a_{0}$ is a positive constant, $k_{s}$ is the rate constant, $n$ is a material constant and $\gamma=\left(\mu+\frac{\kappa}{2}\right) j$ is assumed (for more details, see Ahmadi (1976).

\section{NON-DIMENSIONALIZATION}

Introducing the following non-dimension variables

$$
\begin{gathered}
x=\frac{\bar{x}}{L}, y=\frac{\bar{y}}{L} G r^{1 / 4}, u=\frac{L}{\nu G r^{1 / 2}} \bar{u}, v=\frac{L}{\nu G r^{1 / 4}} \bar{v} \\
\omega=\frac{L^{2}}{\nu G r^{3 / 4}} \bar{\omega}, \theta=\frac{T-T_{\infty}}{T_{f}-T_{\infty}}, h=\frac{\bar{a}}{a_{0}}, h_{1}=\frac{\bar{b}}{a_{0}}
\end{gathered}
$$

where $G r=\frac{g^{*} \beta_{T_{0}}\left(T_{f}-T_{\infty}\right) L^{3}}{\nu^{2}}$ represents the Grashof number.

In view of the continuity equation (1), we introduce the stream function $\psi$ by

$$
u=\frac{\partial \psi}{\partial y}, \quad v=-\frac{\partial \psi}{\partial x}
$$

Using (8) and (9) into (2)-(6), we get the following momentum, angular momentum, energy, and species $\mathrm{A}$ and $\mathrm{B}$ concentration equations

$$
\begin{gathered}
\frac{1}{\epsilon^{2}}\left(\frac{\partial \psi}{\partial y} \frac{\partial^{2} \psi}{\partial x \partial y}-\frac{\partial \psi}{\partial x} \frac{\partial^{2} \psi}{\partial y^{2}}\right)-\frac{1}{\epsilon}\left(\frac{1}{1-N}\right) \frac{\partial^{3} \psi}{\partial y^{3}}-\left(\frac{N}{1-N}\right) \frac{\partial \omega}{\partial y} \\
-\frac{g^{*} \beta_{1}\left(T_{f}-T_{\infty}\right)}{\nu^{2} G r} \theta\left(1+\frac{\beta_{2}}{\beta_{1}} \theta\left(T_{f}-T_{\infty}\right)\right)+\frac{1}{D a G r^{1 / 2}} \frac{\partial \psi}{\partial y}=0 \\
\frac{1}{\epsilon}\left(\frac{\partial \psi}{\partial y} \frac{\partial \omega}{\partial x}-\frac{\partial \psi}{\partial x} \frac{\partial \omega}{\partial y}\right)+\left(\frac{N}{1-N}\right)\left(2 \omega+\frac{1}{\epsilon} \frac{\partial^{2} \psi}{\partial y^{2}}\right)=0 \\
-\left(\frac{2-N}{2-2 N}\right) \frac{\partial^{2} \omega}{\partial y^{2}} \\
\frac{\partial \psi}{\partial y} \frac{\partial \theta}{\partial x}-\frac{\partial \psi}{\partial x} \frac{\partial \theta}{\partial y}-\frac{1}{P r} \frac{\partial^{2} \theta}{\partial y^{2}}=0 \\
\frac{\partial \psi}{\partial y} \frac{\partial \psi}{\partial x} \frac{\partial h_{1}}{\partial x}-\frac{\partial \psi}{\partial x}-\frac{1}{\partial y} \frac{\partial^{2} h}{\partial y^{2}}+K h h_{1}^{2}=0 \\
\frac{\partial h_{1}}{\partial y}-\frac{\delta}{S c} \frac{\partial^{2} h_{1}}{\partial y^{2}}-K h_{1}^{2}=0
\end{gathered}
$$

In usual definitions, $\nu$ represents the kinematic viscosity, $\operatorname{Pr}=\frac{\nu}{\alpha}$ is the Prandtl number, $D a=\frac{K_{p}}{L^{2}}$ indicates the Darcy number, $K=\frac{k_{c} a_{0}{ }^{2} L}{\nu G r^{1 / 2}}$ measure the strength of homogeneous reaction, $S c=\frac{\nu}{D_{A}}$ represents the Schmidt number, $\delta=\frac{D_{B}}{D_{A}}$ is the ratio of diffusion coefficient and $N=\frac{\kappa}{\mu+\kappa}(0 \leq N<1)$ indicates the Coupling number [ Cowin (1968)].

Now the boundary conditions (7) become

$$
\begin{gathered}
\frac{\partial \psi}{\partial y}=0, \quad \frac{\partial \psi}{\partial x}=0, \quad \omega=-n \frac{\partial^{2} \psi}{\partial y^{2}}, \quad \frac{\partial \theta}{\partial y}=-B i(1-\theta), \\
\frac{\partial h}{\partial y}=K_{s} h, \quad \delta \frac{\partial h_{1}}{\partial y}=-K_{s} h \text { at } y=0 \\
\frac{\partial \psi}{\partial y}=0, \quad \omega=0, \quad \theta=0, \quad h=1, \quad h_{1}=0 \quad \text { as } \quad y \rightarrow \infty
\end{gathered}
$$

where $K_{s}=\frac{k_{s} L G r^{-1 / 4}}{D_{A}}$ measures the strength of heterogeneous (surface) reaction. Further, $B i=\frac{h_{f} L}{k G r^{1 / 4}}$ is known as the Biot number, which is the ratio of the internal thermal resistance of the plate to the boundary layer thermal resistance of the hot fluid at the bottom of the surface.

\section{SIMILARITY EQUATIONS VIA LIE GROUP TRANSFORMATIONS}

A one-parameter scaling group of transformations, selected as (Tapanidis et al., 2003)

$$
\begin{gathered}
\Gamma: x^{*}=x e^{\varepsilon \alpha_{1}}, y^{*}=y e^{\varepsilon \alpha_{2}}, \psi^{*}=\psi e^{\varepsilon \alpha_{3}}, \omega^{*}=\omega e^{\varepsilon \alpha_{4}}, \theta^{*}=\theta e^{\varepsilon \alpha_{5}}, \\
h^{*}=h e^{\varepsilon \alpha_{6}}, h_{1}^{*}=h_{1} e^{\varepsilon \alpha_{7}}, \beta_{1}^{*}=\beta_{1} e^{\varepsilon \alpha_{8}}, \beta_{2}^{*}=\beta_{2} e^{\varepsilon \alpha_{9}}
\end{gathered}
$$

Here $\varepsilon \neq 0$ is the parameter of the group and $\alpha^{\prime} s$ are arbitrary real numbers not all simultaneously zero. Equations (10)- (13) along with the boundary conditions (14) do not alter under the group of transformations in Eq.(15) if $\alpha_{i}$ 's hold following relationship

$$
\begin{aligned}
& \alpha_{1}+2 \alpha_{2}-2 \alpha_{3}=3 \alpha_{2}-\alpha_{3}=\alpha_{2}-\alpha_{4}=-\alpha_{5}-\alpha_{8}=-2 \alpha_{5}-\alpha_{9} \\
& \alpha_{1}+\alpha_{2}-\alpha_{3}-\alpha_{4}=2 \alpha_{2}-\alpha_{4}=-\alpha_{4}=2 \alpha_{2}-\alpha_{3} \\
& \alpha_{1}+\alpha_{2}-\alpha_{3}-\alpha_{5}=2 \alpha_{2}-\alpha_{5} ; \alpha_{2}-\alpha_{6}=-\alpha_{6} \\
& \alpha_{1}+\alpha_{2}-\alpha_{3}-\alpha_{6}=2 \alpha_{2}-\alpha_{6}=-\alpha_{6}-2 \alpha_{7} ;-\alpha_{6}=0 \\
& \alpha_{1}+\alpha_{2}-\alpha_{3}-\alpha_{7}=2 \alpha_{2}-\alpha_{7}=-\alpha_{6}-2 \alpha_{7} \\
& \alpha_{2}-\alpha_{7}=-\alpha_{6} \alpha_{2}-\alpha_{5}=0=-\alpha_{5} ;-\alpha_{4}=2 \alpha_{2}-\alpha_{3}
\end{aligned}
$$

Using the procedure explained in the article by (Uddin et al., 2012) and (Mutlag et al., 2013), we have the following similarity transformations:

$$
\begin{gathered}
\eta=y, \psi=x f(\eta), \omega=x g(\eta), \beta_{1}=\beta_{T_{0}} x, \\
\beta_{2}=\beta_{T_{1}} x, \theta=\theta(\eta), h=h(\eta), h_{1}=h_{1}(\eta)
\end{gathered}
$$

where $\beta_{T_{0}}$ and $\beta_{T_{1}}$ are constant coefficients of thermal expansion.

Using Eq. (17) into Eqn. (10)-(13), we get the following similarity equations

$$
\begin{gathered}
\frac{1}{\epsilon}\left(\frac{1}{1-N}\right) f^{\prime \prime \prime}+\frac{1}{\epsilon^{2}} f f^{\prime \prime}-\frac{1}{\epsilon^{2}} f^{\prime 2}+\left(\frac{N}{1-N}\right) g^{\prime} \\
+\theta(1+\chi \theta)-\frac{1}{D a G r^{1 / 2}} f^{\prime}=0 \\
\left(\frac{2-N}{2-2 N}\right) g^{\prime \prime}+\frac{1}{\epsilon} f g^{\prime}-\frac{1}{\epsilon} f^{\prime} g-\left(\frac{N}{1-N}\right)\left(2 g+\frac{1}{\epsilon} f^{\prime \prime}\right)=0 \\
\frac{1}{P r} \theta^{\prime \prime}+f \theta^{\prime}=0
\end{gathered}
$$




$$
\begin{aligned}
& \frac{1}{S c} h^{\prime \prime}+f h^{\prime}-K h h_{1}{ }^{2}=0 \\
& \frac{\delta}{S c} h_{1}^{\prime \prime}+f h_{1}^{\prime}+K h h_{1}{ }^{2}=0
\end{aligned}
$$

where the primes indicate differentiation with respect to $\eta$ alone, $\chi=$ $\frac{\beta_{T_{1}}}{\beta_{T_{0}}}\left(T_{f}-T_{\infty}\right)$ the nonlinear convection (or) nonlinear density temperature (NDT) parameter.

The corresponding boundary conditions (14) are

$$
\begin{gathered}
f(0)=0, f^{\prime}(0)=0, f^{\prime}(\eta)=0 \quad \text { as } \eta \rightarrow \infty \\
g(0)=-n f^{\prime \prime}(0), g(\eta)=0 \quad \text { as } \eta \rightarrow \infty \\
\theta^{\prime}(0)=-B i[1-\theta(0)], \theta(\eta)=0 \quad \text { as } \eta \rightarrow \infty \\
h^{\prime}(0)=K_{s} h(0), h(\infty)=1 \quad \text { as } \eta \rightarrow \infty \\
\delta h_{1}^{\prime}(0)=-K_{s} h(0) h_{1}(\infty)=0 \quad \text { as } \eta \rightarrow \infty .
\end{gathered}
$$

It is predicted that the diffusion coefficients of chemical species A and B are of comparable size, which undergo further assumption that the diffusion coefficients $D_{A}$ and $D_{B}$ are equal, i.e., $\delta=1$ Merkin (1996).

$$
h(\eta)+h_{1}(\eta)=1
$$

Thus, equations (21) and (22) reduce to

$$
\frac{1}{S c} h^{\prime \prime}+f h^{\prime}-K h(1-h)^{2}=0
$$

and are subject to the boundary condition

$$
h^{\prime}(0)=K_{s} h(0), \quad h(\eta)=1 \quad \text { as } \eta \rightarrow \infty
$$

The wall shear stress, wall couple stress and the heat transfer from the plate are

$\tau_{w}=\left[(\mu+\kappa) \frac{\partial \bar{u}}{\partial \bar{y}}+\kappa \bar{\omega}\right]_{\bar{y}=0} m_{w}=\gamma\left[\frac{\partial \bar{\omega}}{\partial \bar{y}}\right]_{\bar{y}=0}$ and $q_{w}=-k\left[\frac{\partial T}{\partial \bar{y}}\right]_{\bar{y}=0}$

The non-dimensional skin friction $C_{f}=\frac{2 \tau_{w}}{\rho \bar{u}_{*}^{2}}$, wall couple stress $M_{w}=$ $\frac{m_{w}}{\rho \bar{u}_{*}^{2} \bar{x}}$, and the local Nusselt number $N u_{\bar{x}}=\frac{q_{w} \bar{x}}{k\left(T_{f}-T_{\infty}\right)}$ are given by

$$
\begin{gathered}
C_{f} G r_{\bar{x}}^{1 / 4}=2\left(\frac{1-n N}{1-N}\right) f^{\prime \prime}(0), M_{w} G r_{\bar{x}}^{1 / 2}=\left(\frac{2-N}{2-2 N}\right) g^{\prime}(0) \\
\text { and } \frac{N u_{\bar{x}}}{G r_{\bar{x}}^{1 / 4}}=-\theta^{\prime}(0) .
\end{gathered}
$$

where $G r_{\bar{x}}=\frac{g^{*} \beta_{T_{0}}\left(T_{f}-T_{\infty}\right) \bar{x}^{3}}{\nu^{2}}$ is the local Grashof number.

\section{NUMERICAL SOLUTION}

Here, the spectral quasi-linearization method (SQLM) is used for solving the non-linear system of Eqs. (18) - (20) and (25) along with the boundary conditions (23(a) - 23(c) and (26)). The Quasi-linearization method is the generalized Newton-Raphson method, introduced by Bellman and Kalaba (1965) for solving nonlinear boundary value problems.

Assume that the solutions $f_{r}, g_{r}, \theta_{r}$ and $h_{r}$ of Eqs. (18)-(20) and (25) at the $(r+1)^{t h}$ iteration are $f_{r+1}, g_{r+1}, \theta_{r+1}$ and $h_{r+1}$. If the solutions at the previous iteration is sufficiently close to the present iteration, the nonlinear components of the Eqs. (18)-(20) and (25) can be linearised using Taylors series expansions for multiple variables and neglecting the second and higher order derivative terms, so the Eqs. (18)-(20) and (25) give iterative sequence of linearized equations in the following form:

$$
\frac{1}{\epsilon}\left(\frac{1}{1-N}\right) f_{r+1}^{\prime \prime \prime}+\frac{1}{\epsilon^{2}} a_{1, r} f_{r+1}^{\prime \prime}+a_{2, r} f_{r+1}^{\prime}+\frac{1}{\epsilon^{2}} a_{3, r} f_{r+1}
$$

$$
\begin{gathered}
+a_{4, r} \theta_{r+1}+\left(\frac{N}{1-N}\right) g_{r+1}^{\prime}=R_{1, r} \\
\left(\frac{2-N}{2-2 N}\right) g_{r+1}^{\prime \prime}+\frac{1}{\epsilon} b_{3, r} g_{r+1}^{\prime}+b_{4, r} g_{r+1}+\frac{1}{\epsilon} b_{1, r} f_{r+1}^{\prime} \\
+\frac{1}{\epsilon} b_{2, r} f_{r+1}-\left(\frac{N}{1-N}\right) f_{r+1}^{\prime \prime}=R_{2, r} \\
\frac{1}{P r} \theta_{r+1}^{\prime \prime}+c_{1, r} f_{r+1}+c_{2, r} \theta_{r+1}^{\prime}=R_{3, r} \\
\frac{1}{S c} h_{r+1}^{\prime \prime}+d_{1, r} f_{r+1}+d_{2, r} h_{r+1}^{\prime}+d_{3, r} h_{r+1}=R_{4, r}
\end{gathered}
$$

Where the coefficients $a_{s 1, r}(s 1=1,2,3,4), b_{s 2, r}(s 2=1,2, . ., 4)$, $c_{s 3, r}(s 3=1,2), d_{s 4, r}(s 4=1,2,3)$ and $R_{s 5, r}(s 5=1,2, . ., 4)$ are known functions calculated from previous iterations and are defined as

$$
\begin{gathered}
a_{1, r}=f_{r}, a_{2, r}=\frac{-2}{\epsilon^{2}} f_{r}^{\prime}-\frac{1}{D a G r^{1 / 2}}, a_{3, r}=f_{r}^{\prime \prime}, a_{4, r}=1+2 \chi \theta_{r}, \\
b_{1, r}=-g_{r}, b_{2, r}=g_{r}^{\prime}, b_{3, r}=f_{r}, b_{4, r}=-\frac{1}{\epsilon} f_{r}^{\prime}-\left(\frac{2 N}{1-N}\right), \\
c_{1, r}=\theta_{r}^{\prime}, c_{2, r}=f_{r}, d_{1, r}=h_{r}^{\prime}, d_{2, r}=f_{r}, d_{3, r}=K\left(4 h_{r}-3 h_{r}^{2}-1\right), \\
R_{1, r}=\frac{1}{\epsilon^{2}} f_{r} f_{r}^{\prime \prime}-\frac{1}{\epsilon^{2}}\left(f_{r}^{\prime}\right)^{2}+\chi\left(\theta_{r}\right)^{2} R_{2, r}=\frac{1}{\epsilon^{2}} f_{r} g_{r}^{\prime}-\frac{1}{\epsilon^{2}} f_{r}^{\prime} g_{r}, \\
R_{3, r}=f_{r} \theta_{r}^{\prime}, R_{4, r}=2 K\left(h_{r}^{2}-h_{r}^{3}\right)+f_{r} h_{r}^{\prime}
\end{gathered}
$$

subject to boundary conditions:

$$
\begin{gathered}
f_{r+1}(0)=0, f_{r+1}^{\prime}(0)=0, g_{r+1}(0)=-n f_{r+1}^{\prime \prime}(0), \\
\theta_{r+1}^{\prime}(0)=-B i(1-\theta(0)), h_{r+1}^{\prime}(0)=K_{s} h(0), \\
f_{r+1}^{\prime}(\infty)=0, g_{r+1}(\infty)=0, \theta_{r+1}(\infty)=0, h_{r+1}(\infty)=1
\end{gathered}
$$

The above linearized system of Eqs. (29) to (32) can be worked iteratively using Chebyshev pseudo-spectral method. [For more details, one can refer the works of (Motsa et al., 2014b,c)]. To start the SQLM scheme for the system of equations (29) - (32), the initial guesses are chosen as functions that satisfy the boundary conditions:

$$
f_{0}(\eta)=0, g_{0}(\eta)=0, \theta_{0}=\frac{B i}{B i+1} e^{-\eta}, h_{0}=1-e^{-\eta K_{s}}
$$

Starting from the set of initial approximations $f_{0}, g_{0}, \theta_{0}, h_{0}$, the iteration schemes (29) to (32) can be worked out iteratively for $f_{r+1}, g_{r+1}$, $\theta_{r+1}, \quad h_{r+1}$ when $\mathrm{r}=0,1,2, \ldots$. To solve equations (29) to (32) discretize the equation using the Chebyshev spectral collocation method after transforming the domain in $\eta$ to the interval $[-1,1]$ where the spectral method can be implemented. For the convenience of the numerical computations, the physical region is approximated by truncated domain $\left[0, \eta_{\infty}\right]$, where $\eta_{\infty}$ is a finite length chosen to be large enough to represent the flow properties behaviour when $\eta$ is large. Use the transformation $\eta=\eta_{\infty}(\tau+1) / 2$ to map the interval $\left[0, \eta_{\infty}\right]$ to $[-1,1]$.

The basic idea behind the spectral collocation method is the first appearance of a differentiation matrix $D$ which is applied to approximate the differential coefficients of the unknown variables, for example, $f(\eta)$ at the collocation points as the matrix vector product

$$
\frac{d f}{d \eta}=\sum_{k=0}^{\bar{N}} D_{l k} f\left(\tau_{k}\right)=\mathbf{D F}, l=0,1, \ldots, \bar{N}
$$

The unknown functions are approximated by the Chebyshev interpolating polynomials in such way that they are collocated at the Gauss-Lobatto collocation points defined as

$$
\tau_{j}=\cos \frac{\pi j}{\bar{N}}, \quad j=0,1,2, \ldots, \bar{N},
$$


where $\bar{N}+1$ is the number of collocation points (grid points), $\mathbf{D}=\frac{2 \mathcal{D}}{\eta_{\infty}}$ is the differentiation matrix and its entries are clearly defined in (Canuto et al., 2006), and $\mathbf{F}=\left[f\left(\tau_{0}\right), f\left(\tau_{1}\right), \ldots, f\left(\tau_{\bar{N}}\right)\right]^{T}$ is the vector function at the collocation points. Similar vector functions corresponding to $g, \theta$ and $h$ are denoted by $\mathbf{G}, \Theta$ and $\mathbf{H}$ respectively. Higher order derivatives are obtained as powers of $\mathbf{D}$, that is

$$
f^{(q)}=\mathbf{D}^{q} \mathbf{F}, g^{(q)}=\mathbf{D}^{q} \mathbf{G}, \theta^{(q)}=\mathbf{D}^{q} \Theta, h^{(q)}=\mathbf{D}^{q} \mathbf{H} .
$$

where $q$ represents derivatives order. Substituting Eqs.(34)-(36) into Eqs. (29)-(32) leads to the matrix equation

$$
\left[\begin{array}{llll}
A_{11} & A_{12} & A_{13} & A_{14} \\
A_{21} & A_{22} & A_{23} & A_{24} \\
A_{31} & A_{32} & A_{33} & A_{34} \\
A_{41} & A_{42} & A_{43} & A_{44}
\end{array}\right]\left[\begin{array}{c}
\mathbf{F}_{r+1} \\
\mathbf{G}_{r+1} \\
\Theta_{r+1} \\
\mathbf{H}_{r+1}
\end{array}\right]=\left[\begin{array}{l}
\mathbf{R}_{1} \\
\mathbf{R}_{2} \\
\mathbf{R}_{3} \\
\mathbf{R}_{4}
\end{array}\right]
$$

Where

$$
\begin{gathered}
A_{11}=\frac{1}{\epsilon}\left(\frac{1}{1-N}\right) \mathbf{D}^{3}+\frac{1}{\epsilon^{2}} \operatorname{diag}\left[a_{1, r}\right] \mathbf{D}^{2}+\operatorname{diag}\left[a_{2, r}\right] \mathbf{D}+\frac{1}{\epsilon^{2}} \operatorname{diag}\left[a_{3, r}\right], \\
A_{12}=\left(\frac{N}{1-N}\right) \mathbf{D}, A_{13}=\operatorname{diag}\left[a_{4, r}\right] \mathbf{I}, A_{14}=\mathbf{0} \\
A_{21}=\frac{1}{\epsilon}\left(\frac{N}{N-1}\right) \mathbf{D}^{2}+\operatorname{diag}\left[b_{1, r}\right] \mathbf{D}+\frac{1}{\epsilon} \operatorname{diag}\left[b_{2, r}\right], \\
A_{22}=\left(\frac{2-N}{2-2 N}\right) \mathbf{D}^{2}+\frac{1}{\epsilon} \operatorname{diag}\left[b_{3, r}\right] \mathbf{D}+\operatorname{diag}\left[b_{4, r}\right], A_{23}=\mathbf{0}, A_{24}=\mathbf{0} \\
A_{31}=\operatorname{diag}\left[c_{1, r}\right], A_{32}=\mathbf{0}, A_{33}=\frac{1}{P r} \mathbf{D}^{2}+\operatorname{diag}\left[c_{2, r}\right] \mathbf{D}, A_{34}=\mathbf{0} \\
A_{41}=\operatorname{diag}\left[d_{1, r}\right], A_{42}=\mathbf{0}, A_{43}=\mathbf{0}, \\
A_{44}=\frac{1}{S c} \mathbf{D}^{2}+\operatorname{diag}\left[d_{2, r}\right] \mathbf{D}+\operatorname{diag}\left[d_{3, r}\right] \\
\mathbf{R}_{1}=R_{1, r}, \quad \mathbf{R}_{2}=R_{2, r}, \quad \mathbf{R}_{3}=R_{3, r}, \quad \mathbf{R}_{4}=R_{4, r}
\end{gathered}
$$

where $\mathbf{I}$ is an identity matrix, $\mathbf{0}$ is a zero matrix and $\operatorname{diag}[\mathrm{]}$ is a diagonal matrix, all of size $(\bar{N}+1) \times(\bar{N}+1)$. F, G, $\Theta$ and $\mathbf{H}$ are the values of the functions $f, g, \theta$ and $h$ when evaluated at the grid points.

\section{RESULTS AND DISCUSSIONS}

The influence of homogeneous and heterogeneous reactions and nonlinear convection on micropolar fluid flow along a vertical plate embedded in Darcy porous medium with convective boundary condition is analyzed in this paper. In order to study the effects of nonlinear convection parameter $\chi$, Darcy number $D a$, Biot number $B i$, strength of homogeneous and heterogeneous reaction parameters $K$ and $K_{s}$, computations have been carried out in the cases of $n=0, \epsilon=0.6, G r=10, \operatorname{Pr}=0.71$ and $S c=0.22$. The results of the current problem have been compared with that of the results attained by (Merkin, 1976; Nazar et al., 2002; Molla et al., 2006) as a special case by taking $N=0, \epsilon=1, n=0, \operatorname{Pr}=1$, $\chi=0, D a \rightarrow \infty$ and $B i \rightarrow \infty$, and seems to be good agreement in the above case, as presented in Tab. (1). Also, the comparison of $-\theta^{\prime}(0)$ has been made with the results obtained by (Nazar et al., 2002) as shown in Tab. (1) when $n=0.5, \epsilon=1, \operatorname{Pr}=1, B i \rightarrow \infty, D a \rightarrow \infty$ and $\chi=0$.
Table 1 Comparison of $-\theta^{\prime}(0)$ for free convection along a vertical flat plate in Newtonian fluid when $N=0, D a \rightarrow \infty, n=0, \operatorname{Pr}=$ $1, \chi=0, \epsilon=1$ and $B i \rightarrow \infty$.

\begin{tabular}{|c|c|c|c|}
\hline Merkin (1976) & (Nazar et al., 2002) & (Molla et al., 2006) & Present \\
\hline 0.4214 & 0.4214 & 0.4214 & 0.4214313 \\
\hline
\end{tabular}

Table 2 Comparison of $-\theta^{\prime}(0)$ for free convection flow in a micropolar fluid obtained by (Nazar et al., 2002) when $n=0.5, \epsilon=1$, $\operatorname{Pr}=1, \chi=0, D a \rightarrow \infty$ and $B i \rightarrow \infty$.

\begin{tabular}{|l|c|c|}
\hline$N$ & (Nazar et al., 2002$)$ & Present \\
\hline 0.00 & 0.4214 & 0.4214 \\
0.33 & 0.3991 & 0.3990 \\
0.50 & 0.3834 & 0.3834 \\
0.60 & 0.3709 & 0.3709 \\
0.66 & 0.3608 & 0.3608 \\
0.71 & 0.3522 & 0.3522 \\
0.75 & 0.3447 & 0.3447 \\
\hline
\end{tabular}

\subsection{Boundary layer distribution of velocity, microrotation, temperature and species concentration}

\section{a) With varying values of Biot bumber $(B i)$}

The first set of Figs. 2(a) -2 (d) depicts for $N=0.5, D a=1$, $K=1, K_{s}=0.5$ and $\chi=1.0$ and refer to the variation of nondimensional velocity $f^{\prime}$, microrotation $g$, temperature $\theta$ and species concentration $h$ across the boundary layer. When the plate is totally insulated (i,e., $B i=0$ ), the internal thermal resistance of the plate is extremely high and no convective heat transfer to the cold fluid on the upper part of the plate. Fig. 2(a) reads the fluid velocity profiles for different values of the Biot number with fixed values of other parameters. Specifically, at the surface of the plate, the fluid velocity is zero and enhances moderately away from the plate to the free stream value satisfying the boundary conditions. It is interesting to reveal that in momentum boundary layer the fluid velocity enhances with an increase in intensity of convective surface heat transfer $B i$. From Fig. 2(b) it is clear that the microration profile shows reverse rotation near the two boundaries. Fig. 2(c) depicts that, the temperature of fluid is maximum at plate surface and diminishes exponentially to zero far away from the plate. Comparatively, internal thermal resistance of the plate is more for high Biot number than the boundary layer thermal resistance. From Fig. 2(d) it notice that the species concentration increases with increase in Biot number. b) With varying values of nonlinear convection parameter (NDT)

The second set of Figs. 3(a)-3(d) plotted for different values of nonlinear convection parameter on non-dimensional velocity $f^{\prime}$, microrotation $g$, temperature $\theta$ and species concentration $h$ with fixed values of other parameters $N=0.5, D a=1, K=1, K_{s}=0.5$ and $B i=1$. The nonlinear convection parameter $\chi$ measures the nonlinearity in densitytemperature relationship. The influence of nonlinear convection parameter $\chi$ on the velocity profile is depicted in Fig. 3(a). With an increase in $\chi$, the velocity increases, but far away from the plate it shows opposite trend. From Fig. 3(b), it observes that the microrotation is showing reverse rotation near the two boundaries with increased value of nonlinear convection parameter. If $\chi>0$ implies that $T_{f}>T_{\infty}$ hence there is supply of heat from wall to flow region. Similarly $\chi<0$ implies that $T_{f}<T_{\infty}$, heat transfer from fluid to the wall. From Fig. 3(c) it is perceived that the temperature of the fluid reduces with an increase in nonlinear convection parameter. The species concentration enhances with an increase in nonlinear convection parameter as shown in 3(d). c) With Varying values of Darcy number $(D a)$ 


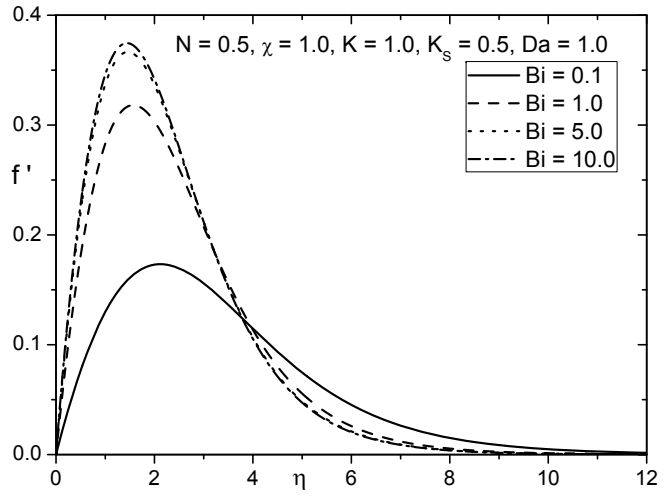

(a)

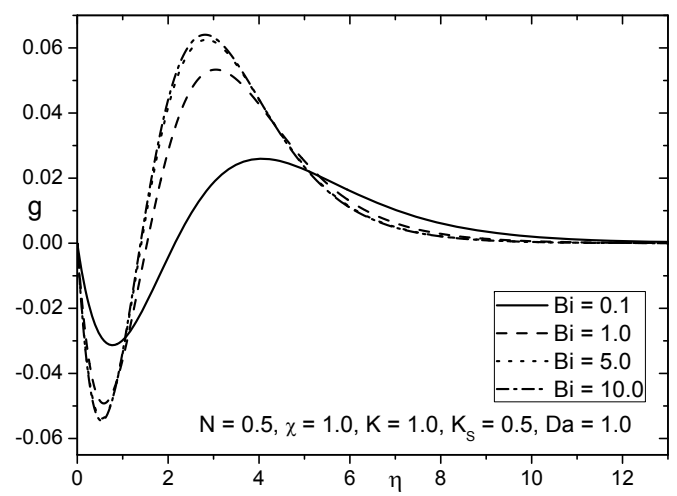

(b)

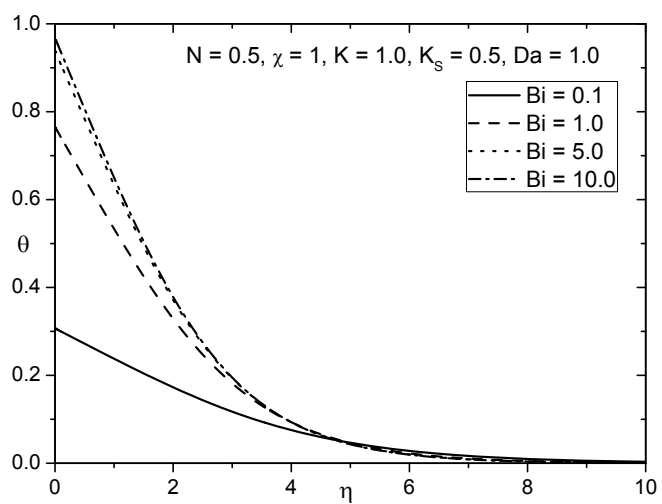

(c)

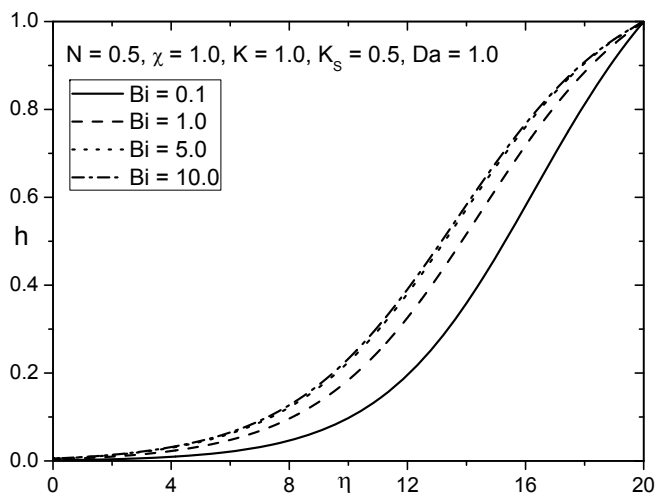

(d)

Fig. 2 Effect of $B i$ on (a) Velocity, (b) Microrotation, (c) Temperature, and (d) Concentration profiles.

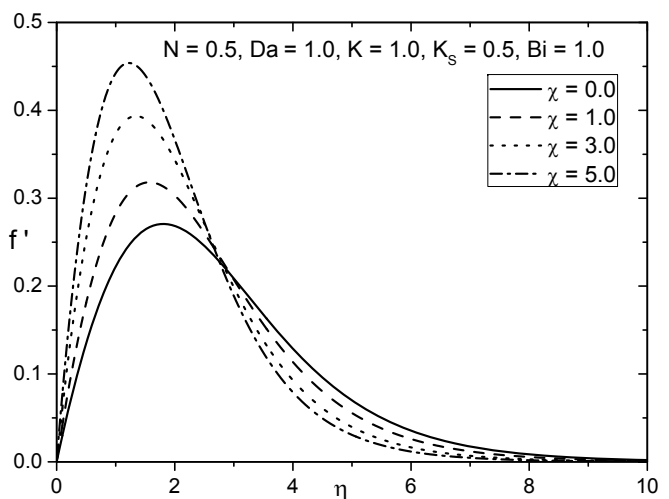

(a)

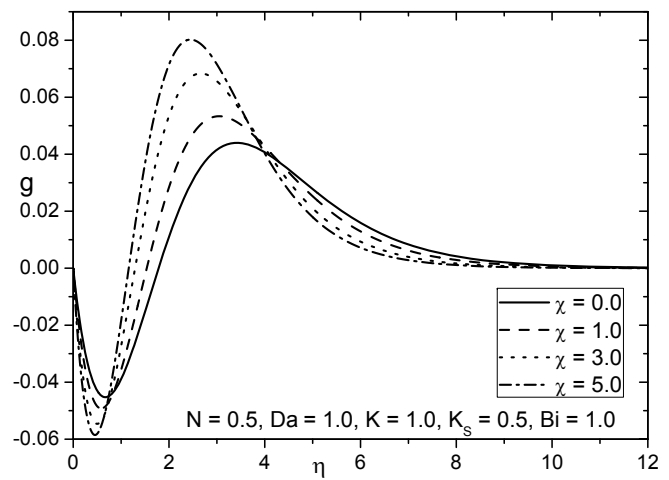

(b)

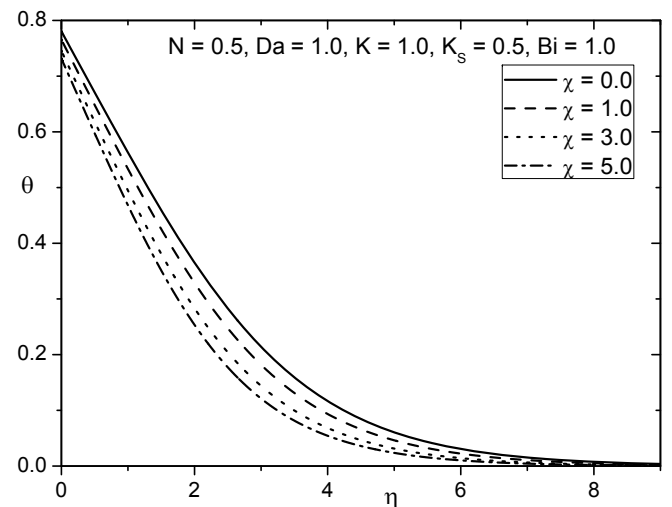

(c)

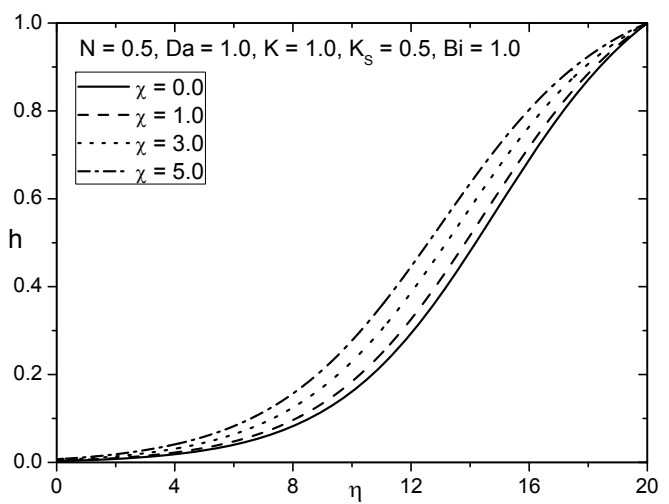

(d)

Fig. 3 Effect of $\chi$ on (a) Velocity, (b) Microrotation, (c) Temperature, and (d) Concentration profiles. 
Table 3 Effects of skin friction, wall couple stress and heat transfer rate for varying values of micropolar parameter $N$, Biot numbers $B i$, nonlinear convection parameter $\chi$ and Darcy number $D a$ with $K=1$ and $K_{s}=0.5$.

\begin{tabular}{|lccc|ccr|}
\hline$N$ & $B i$ & $\chi$ & $D a$ & $C_{f} G r_{\bar{x}}^{1 / 4}$ & $M_{w} G r_{\bar{x}}^{1 / 2}$ & $\frac{N u_{\bar{x}}}{G r_{\bar{x}}^{1 / 4}}$ \\
\hline 0 & 1.0 & 1.0 & 1 & 1.537527 & 0.000000 & 0.305228 \\
0.3 & 1 & 1.0 & 1 & 1.750517 & -0.142651 & 0.294051 \\
0.5 & 1 & 1.0 & 1 & 1.959351 & -0.291564 & 0.283342 \\
0.8 & 1 & 1.0 & 1 & 2.57905 & -0.710582 & 0.253065 \\
\hline 0.5 & 0.1 & 1.0 & 1.0 & 0.649682 & -0.127284 & 0.067754 \\
0.5 & 1 & 1.0 & 1.0 & 1.593042 & -0.251223 & 0.219462 \\
0.5 & 5 & 1.0 & 1.0 & 1.959351 & -0.291564 & 0.283342 \\
0.5 & 10 & 1.0 & 1.0 & 2.022233 & -0.29819 & 0.294465 \\
\hline 0.5 & 1.0 & 0 & 1.0 & 1.106618 & -0.198623 & 0.20424 \\
0.5 & 1.0 & 1 & 1.0 & 1.593042 & -0.251223 & 0.219462 \\
0.5 & 1.0 & 3 & 1.0 & 2.399576 & -0.328708 & 0.239398 \\
0.5 & 1.0 & 5 & 1.0 & 3.086638 & -0.388064 & 0.253076 \\
\hline 0.5 & 5.0 & 1.0 & 0.05 & 1.283075 & -0.156822 & 0.197751 \\
0.5 & 5.0 & 1.0 & 0.1 & 1.530281 & -0.204481 & 0.231549 \\
0.5 & 5.0 & 1.0 & 0.3 & 1.813237 & -0.261512 & 0.266797 \\
0.5 & 5.0 & 1.0 & 0.5 & 1.892408 & -0.277765 & 0.275907 \\
\hline
\end{tabular}

The third set of Figs. 4(a)-4(d) presents the influence of Darcy number on non-dimensional velocity, microrotation, temperature and species concentration with fixed values of other parameter $N=0.5, D a=1$, $K=1, K_{s}=0.5$ and $B i=5$. From Fig. 4(a), it notices that with increase in Darcy number the velocity of the fluid enhances near the wall and farther from the plate it shows opposite trend. Increase in Darcy number means relatively there is rise in permeability $K_{p}$. The porous matrix structure becomes less and less prominent with increasing of permeability. As Darcy number tends to infinity (i,e., $D a \rightarrow \infty$ ) and porosity $\epsilon=1$, the present problem reduces to the free convection flow of a micropolar fluid with a convective boundary condition. Fig. 4(b) reveals that the microrotation show opposite trends within the boundary. From Figs. 4(c) and 4(d) it observes that the temperature and species concentration of the fluid show a reverse trend with an increase in Darcy number. d) With Varying values of homogeneous and heterogeneous reactions ( $K$ and $\left.K_{s}\right)$

The fourth and fifth set of Figs. 5(a)-5(d) refer to the variation of strength of homogeneous and heterogeneous reactions parameter on species concentration $h$ and the rate of mass transfer across the boundary layer for fixed values of other parameter. As an increase in value of $K$ corresponds to increase in the strength of homogeneous reaction rate. From Figs. 5(a) and 5(b) depict that an increase in homogeneous reaction causes decrease in species concentration and rate of mass transfer. As the heterogeneous reaction parameter $K_{s}$ increases, then the strength of heterogeneous reaction rate increases. It is clear from Figs. 5(c) and 5(d) that the species concentration and the rate of mass transfer decreases with the increase of heterogeneous reaction parameter $K_{s}$. And also notice that the influence of heterogeneous reaction is more about the species concentration as compared with the homogeneous reaction.

\subsection{Skin friction, Wall couple stress and heat transfer coefficient}

Table. (3) displays the variations of $C_{f} G r_{\bar{x}}^{1 / 4}, M_{w} G r_{\bar{x}}^{1 / 2}$, and $\frac{N u_{\bar{x}}}{G r_{\bar{x}}^{1 / 4}}$ which are proportional to the coefficients of skin-friction, wall couple stress and heat transfer rate with combined effects of the coupling number $N$, Biot number $B i$, nonlinear convection parameter $\chi$ and Darcy number $D a$ for fixed $K$ and $K_{s}$ parameters. The effect of coupling number $N$ for fixed $B i=5, \chi=1.0$ and $D a=1$, is shown in Tab.(3). Generally, the

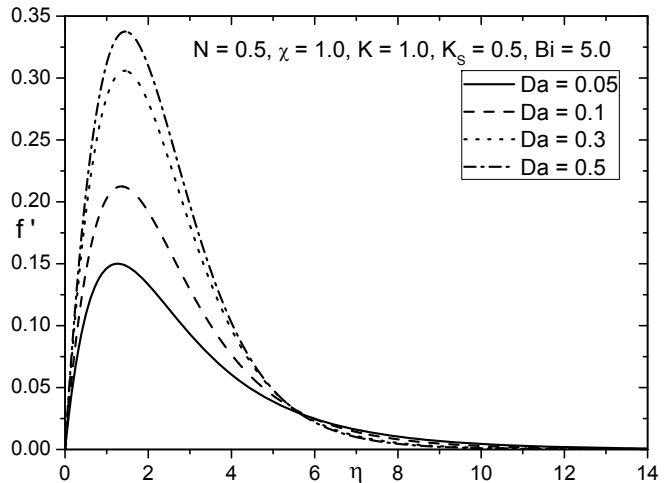

(a)

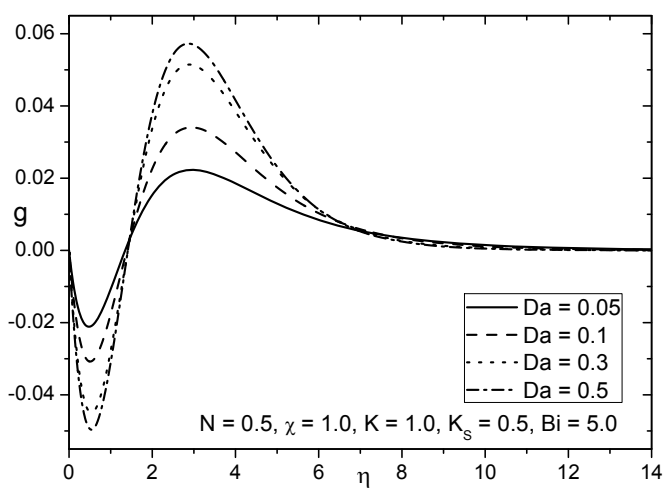

(b)

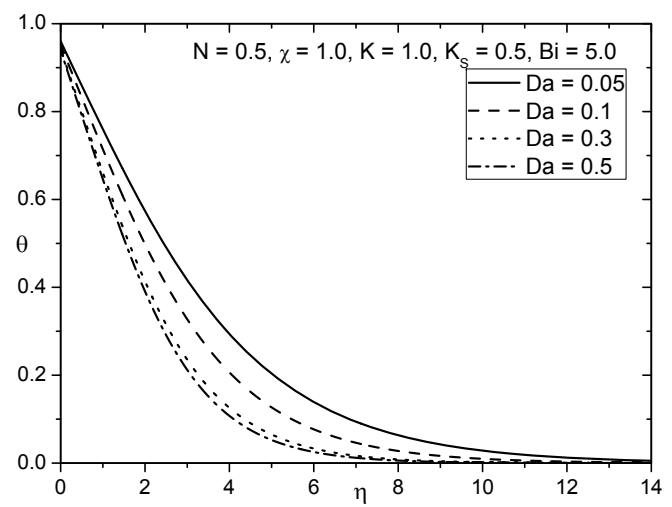

(c)

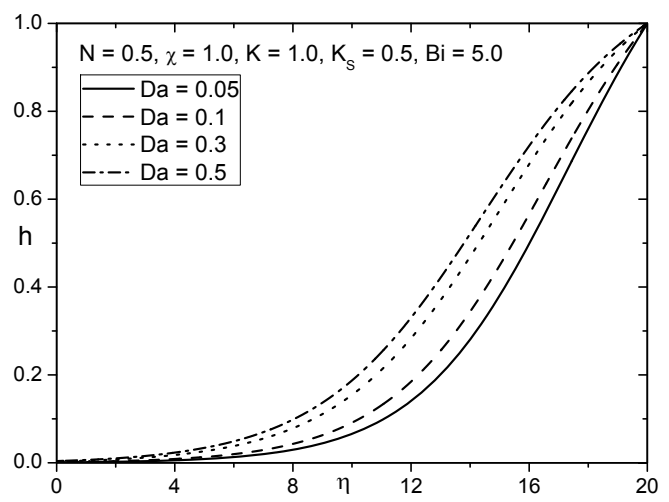

(d)

Fig. 4 Effect of $D a$ on (a) Velocity, (b) Microrotation, (c) Temperature, and (d) Concentration profiles. 


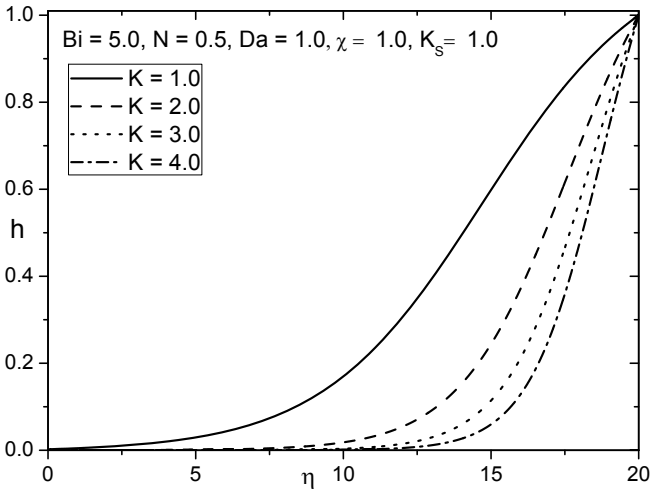

(a)

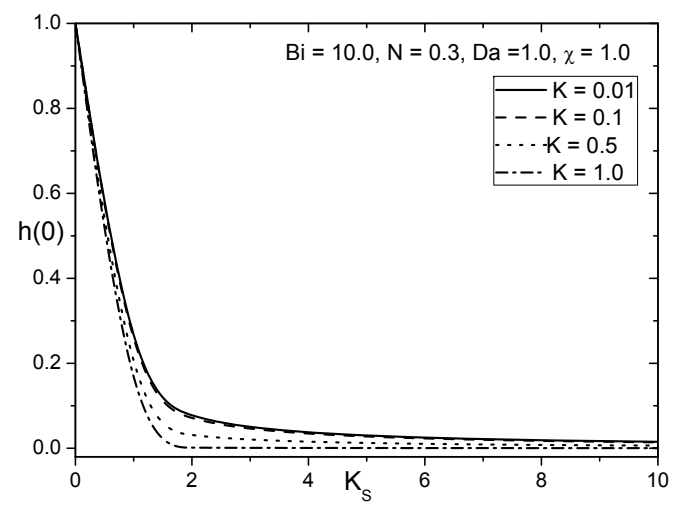

(b)

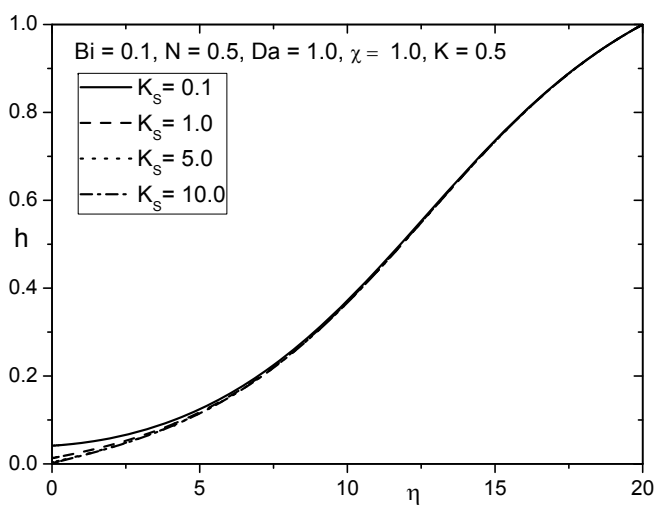

(c)

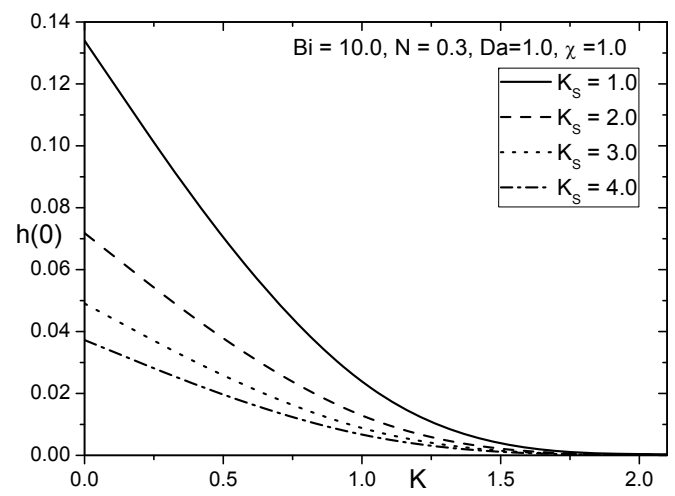

(d)

Fig. 5 Effect of $K$ on (a) Concentration profile $h$, (b) mass transfer rate $h(0)$, Effect of $K_{s}$ on (a) Concentration profile $h$, (b) mass transfer rate $h(0)$. skin friction of micropolar fluid is larger than the viscous fluids $(N=0)$. Since micropolar fluids offer a heavy resistance (resulting from vortex viscosity) to the fluid movement and causes larger skin friction factor compared to viscous fluid. From Tab. (3) it observes that by enhancing the coupling number $N$, increases the skin friction, but reduce the wall couple stress and heat transfer rate. Also for fixed $N=0.5, \chi=1.0$ and $D a=1.0$, the skin-friction as well as heat transfer rate increase, whereas wall couple stress diminishes with the increasing value of Biot number $B i$. From Tab. (3) it is noticed that with the increase of nonlinear convection parameter for fixed parameters $N=0.5, B i=1$ and $D a=$ 1 , both skin friction and heat transfer rate rises and wall couple stress reduces. It is clear from the table that the skin friction and heat transfer rate increases and wall couple stress decreases with an increase of the Darcy number for fixed values of $N=0.5, B i=5$ and $\chi=1.0$.

\section{CONCLUSIONS}

This paper analyzes the effects of nonlinear convection and homogeneousheterogeneous reactions on micropolar fluid flow along a vertical plate in a saturated porous medium with the convective boundary condition. The resulting equations are solved numerically by spectral quasi-linearization method. The main findings are summarized as follows:

- It is observed that an enhancement in Biot number $B i$ leads to reduce the wall couple stress coefficient and enhance the skin friction, heat transfer rate, temperature distribution and species concentration. Moreover, velocity rises near the plate, but shows the reverse behavior far away from the plate. It noticed that the microrotation profile depicts reverse rotation near two boundaries within the boundary layer.

- In the presence of nonlinear convection parameter $\chi$, it is concluded that less temperature and wall couple stress coefficient, but more velocity, species concentration, skin friction and heat transfer rate. Further, microrotation shows opposite trend far away from the wall.

- It is found that the skin friction, heat transfer rate, species concentration and velocity near the plate are increasing, whereas the wall couple stress and temperature are decreasing with the influence of Darcy number.

- It is clear that with an increase in strength of homogeneous and heterogeneous reaction parameters $K$ and $K_{s}$, species concentration and mass transfer rate decrease. And also noticed that the influence of heterogeneous reaction is more at species concentration as compared with homogeneous reaction.

\section{REFERENCES}

Ahmadi, G., 1976, "Self-Similar Solution of Incompressible Micropolar Boundary Layer Flow over a Semi-Infinite Plate," Int J Eng Sci, 14, 639_ 646.

http://dx.doi.org/10.1016/0020-7225(76)90006-9.

Barrow, H., and Rao, T.L.S., 1971, "The Effect of Variable Beta on Free Convection," Br Chem Eng, 16, 704-709.

Beg, O.A., Bhargava, R., Rawat, S., Takhar, H.S., and Beg, T.A., 2007, "Numerical Analysis of Grashof and Darcy Number Effects on Dissipative Natural Convection Boundary Layers in Micropolar Fluid-Saturated Geological Porous Medium," International Journal of Fluid Mechanics Research, 34, 287-307.

http://dx.doi.org/10.1615/InterJFluidMechRes.v34.i4.10.

Bellman, R.E., and Kalaba, R.E., 1965, Quasilinearisation and NonLinear Boundary-Value Problems, Elsevier, New York. 
Canuto, C., Hussaini, M.Y., Quarteroni, A., and Zang, T.A., 2006, Spectral Methods Fundamentals in Single Domains, Scientific Computation, Springer, Berlin, Germany.

Chamka, A.J., Rashad, A.M., Ramreddy, C., and Murthy, P.V.S.N., 2014, "Viscous Dissipation and Magnetic Field Effects in a Non-Darcy Porous Medium Saturated with a Nanofluid under Convective Boundary Condition," Special Topics and Reviews in Porous Media-An International Journal, 5(1), 27-39.

http://dx.doi.org/10.1615/SpecialTopicsRevPorousMedia.v5.i1.30.

Cowin, S., 1968, "Polar Fluids," Physics of Fluids, 11, 1919-1927. http://dx.doi.org/10.1063/1.1692219.

El-Kabeir, S.M.M., and Gorla, R.S.R., 2007, "MHD Effects on Natural Convection in a Micropolar Fluid at a Three-Dimensional Stagnation Point in a Porous Medium," International Journal of Fluid Mechanics Research, 34(2), 145-158.

http://dx.doi.org/10.1615/InterJFluidMechRes.v34.i2.40.

Eremeyev, V., Lebedev, L., and Altenbach, H., 2013, Foundations of Micropolar Mechanics, Springer, New York.

Eringen, A., 1966, "Theory of Micropolar Fluids," J Math and Mech, 16, $1-18$.

http://dx.doi.org/10.1512/iumj.1967.16.16001.

Gangadhar, K., Reddy, N., and Kameswaran, P., 2012, "Similarity Solution of Hydro Magnetic Heat and Mass Transfer over a Vertical Plate with Convective Surface Boundary Condition and Chemical Reaction," International Journal of Nonlinear Science, 13(3), 298-307.

Hayat, T., Farooq, M., and Alsaedi, A., 2015a, "HomogeneousHeterogeneous Reactions in the Stagnation Point Flow of Carbon Nanotubes with Newtonian Heating," AIP Advances, 5(2), 027130-1-18. http://dx.doi.org/10.1063/1.4908602.

Hayat, T., Khan, M., Farooq, M., Yasmeen, T., and Alsaedi, A., 2016a, "Stagnation Point Flow with Cattaneo-Christov Heat Flux and Homogeneous-Heterogeneous Reactions," Journal of Molecular Liquids, 220, 49-55.

http://dx.doi.org/10.1016/j.molliq.2016.04.032.

Hayat, T., Waqas, M., Shehzad, S., and Alsaedi, A., 2015b, "MHD Stagnation Point Flow of Jeffrey Fluid by a Radially Stretching Surface with Viscous Dissipation and Joule Heating," J Hydrol Hydromech, 63(2), 311-317.

http://dx.doi.org/10.1515/johh-2015-0038.

Hayat, T., Waqas, M., Shehzad, S., and Alsaedi, A., 2016b, "Chemically Reactive Flow of Third Grade Fluid by an Exponentially Convected Stretching Sheet," Journal of Molecular Liquids, 223, 853-860. http://dx.doi.org/10.1016/j.molliq.2016.09.007.

Hayat, T., Waqas, M., Shehzad, S., and Alsaedi, A., 2016c, "On Model of Burgers Fluid Subject to Magneto Nanoparticles and Convective Conditions," Journal of Molecular Liquids, 221, 181-187.

http://dx.doi.org/10.1016/j.molliq.2016.06.087.

Hayat, T., Waqas, M., Shehzad, S., and Alsaedi, A., 2016d, "Stretched Flow of Carreau Nanofluid with Convective Boundary Condition," Pramana Journal of Physics, 86, 3-17.

http://dx.doi.org/10.1007/s12043-015-1137-y.

Hayat, T., Waqas, M., Shehzadand, S., and Alsaedi, A., 2013, "Mixed Convection Radiative Flow of Maxwell Fluid near a Stagnation Point with Convective Condition," Journal of Mechanics, 29(3), 403-409.

http://dx.doi.org/10.1017/jmech.2013.6.
Hayat, T., Waqas, M., Shehzadand, S., and Alsaedi, A., 2014, "Effects of Joule heating and thermophoresis on stretched flow with convective boundary conditions," Scientia Iranica B, 21(3), 682-692.

Ingham, D.B., and Pop, I., 2005, Transport Phenomena in Porous Media III, Elsevier, Oxford.

Kameswaran, P.K., Shaw, S., Sibanda, P., and Murthy, P.V.S.N., 2013, "HomogeneousâĂŞHeterogeneous Reactions in a Nanofluid Flow due to a Porous Stretching Sheet," International Journal of Heat and Mass Transfer, 57(2), 465-472.

http://dx.doi.org/10.1016/j.ijheatmasstransfer.2012.10.047.

Kameswaran, P.K., Sibanda, P., Partha, M.K., and Murthy, P.V.S.N., 2014, "Thermophoretic and Nonlinear Convection in Non-Darcy Porous Medium," Journal of Heat Transfer, 136(4), 042601-1-9.

http://dx.doi.org/10.1115/1.4025902.

Lukaszewicz, G., 1999, Micropolar fluids - Theory and Applications, Springer, Birkhaauser, Basel, Switzerland.

Merkin, J.H., 1996, "A Model for Isothermal HomogeneousHeterogeneous Reactions in Boundary Layer Flow," Mathematical and Computer Modelling, 24(8), 125-136. http://dx.doi.org/10.1016/0895-7177(96)00145-8.

Merkin, J., 1976, "Free Convection Boundary Layer on an Isothermal Horizontal Circular Cylinders," Proceedings of ASME/AIChE, Heat Transfer Conference, 911, St. Louis, Mo, USA.

Molla, M.M., Hossain, M.A., and Paul, M.C., 2006, "Natural Convection Flow from an Isothermal Horizontal Circular Cylinder in Presence of Heat Generation," International Journal of Engineering Science, 44, 949-958.

http://dx.doi.org/10.1016/j.ijengsci.2006.05.002.

Motsa, S.S., Awad, F.G., Makukula, Z.G., and Sibanda, P., 2014a, "The Spectral Homotopy Analysis Method Extended to Systems of Partial Differential Equations," Abstract and Applied Analysis, 2014, 1-11. http://dx.doi.org/10.1155/2014/241594.

Motsa, S.S., Dlamini, P.G., and Khumalo, M., 2014b, "Spectral Relaxation Method and Spectral Quasilinearization Method for Solving Unsteady Boundary Layer Flow Problems," Advances in Mathematical Physics, 2014, 1-12.

http://dx.doi.org/10.1155/2014/341964.

Motsa, S.S., Sibanda, P., Ngnotchouye, J.M., and Marewo, G.T., 2014c, "A Spectral Relaxation Approach for Unsteady Boundary-Layer Flow and Heat Transfer of a Nanofluid over a Permeable Stretching/Shrinking Sheet," Advances in Mathematical Physics, 2014, 1-10. http://dx.doi.org/10.1155/2014/564942.

Mutlag, A.A., Uddin, M.J., Hamad, M.A.A., and Ismail, A.I.M., 2013, "Heat Transfer Analysis for Falkner-Skan Boundary Layer Flow Past a Stationary Wedge with Slips Boundary Conditions Considering Temperature-Dependent Thermal Conductivity," Sains Malaysiana, 42(6), 855-862.

Nandkeolyar, R., Kameswaran, P.K., Shaw, S., and Sibanda, P., 2014, "Heat Transfer on Nanofluid Flow with Homogeneous-Heterogeneous Reactions and Internal Heat Generation," Journal of Heat Transfer, 136, 122001-1-8.

http://dx.doi.org/10.1115/1.4028644.

Nazar, R., Amin, N., and Pop, I., 2002, "Free Convection Boundary Layer on an Isothermal Horizontal Circular Cylinder in a Micropolar Fluid," Proceedings of the 12th International Heat Transfer Conference, 2, 525530, Elsevier, Paris, France. 
Nield, D.A., and Bejan, A., 2013, Convection in Porous Media, Springer.

Pal, D., and Chatterjee, S., 2015, "Effects of Radiation on DarcyForchheimer Convective Flow over a Stretching Sheet in a Micropolar Fluid with Non-Uniform Heat Source/Sink," Journal of Applied Fluid Mechanics, 8(2), 207-212.

Partha, M.K., 2010, "Nonlinear Convection in a Non-Darcy Porous Medium," Applied Mathematics and Mechanics, 31, 565-574.

http://dx.doi.org/10.1007/s10483-010-0504-6.

RamReddy, C., Pradeepa, T., and Srinivasacharya, D., 2015, "Similarity Solution for Free Convection Flow of a Micropolar Fluid under Convective Boundary Condition Via Lie Scaling Group Transformations," Advances in High Energy Physics, 2015, 1-16.

http://dx.doi.org//10.1155/2015/650813.

Shaw, S., Kameswaran, P.K., and Sibanda, P., 2013, "HomogeneousHeterogeneous Reactions in Micropolar Fluid Flow from a Permeable Stretching or Shrinking Sheet in a Porous Medium," Boundary Value Problems, 2013, 77 .

http://dx.doi.org/10.1186/1687-2770-2013-77.

Shaw, S., Kameswaran, P.K., and Sibanda, P., 2016, "Effects Of Slip on Nonlinear Convection in Nanofluid Flow on Stretching Surfaces," Boundary Value Problems, 2016(2).

http://dx.doi.org/10.1186/s13661-015-0506-2.

Shehzad1, S., Waqas, M., Alsaedi, A., and Hayat, T., 2016, "Flow and Heat Transfer over an Unsteady Stretching Sheet in a Micropolar Fluid with Convective Boundary Condition," Journal of Applied Fluid Mechanics, 9, 1437-1445.
Singh, A., 2013, "Numerical Solution of Free Convection Flow of a Micro-Polar Fluid Past a Porous Vertical Plate," Indian J Pure Appl Phys, 41, 936-940.

Srinivasacharya, D., and RamReddy, C., 2013, "Free Convective Heat and Mass Transfer in a Doubly Stratified Non-Darcy Micropolar Fluid," Korean Journal of Chemical Engineering, 28(9), 1824-1832. http://dx.doi.org/10.1007/s11814-011-0069-6.

Tapanidis, T., Tsagas, G., and Mazumdar, H.P., 2003, "Application of Scaling Group of Transformations to Viscoelastic Second-Grade Fluid Flow," Nonlinear Functional Analysis and Applications, 8(3), 345-350.

Uddin, M.J., Khan, W.A., and Ismail, A.I.M., 2012, “MHD Free Convective Boundary Layer Flow of a Nanofluid Past a Flat Vertical Plate with Newtonian Heating Boundary Condition," Plos One, 7, e49499. http://dx.doi.org/10.1371/journal.pone.0049499.

Vafai, K., 2000, Handbook of Porous Media, Marcel Dekker, New York.

Waqas, M., Farooq, M., Khan, M., Alsaedi, A., Hayat, T., and Yasmeen, T., 2016, "Magnetohydrodynamic Mixed Convection Flow of Micropolar Liquid due to Nonlinear Stretched Sheet with Convective Condition," International Journal of Heat and Mass Transfer, 102, 766-772. http://http://dx.doi.org/10.1016/j.ijheatmasstransfer.2016.05.142.

Yacob, N.A., and Ishak, A., 2012, "Stagnation Point Flow Towards Stretching/Shrinking Sheet in a Micropolar Fluid with a Convective Boundary Condition," Canadian Journal of Chemical Engineering, 90, 621-626.

http://dx.doi.org/10.1002/cjce.20517. 